\title{
NGHIÊN CÚU PHƯƠNG PHÁP KIỂM ĐỊNH MÁY THU GNSS DỰA TRÊN GIẢI PHÁP MÔ PHỎNG TÍN HIẸU
}

\author{
BÙI ĐĂNG QUANG ${ }^{(1)}$, NGUYẼ̃N ĐİNH THUẬN ${ }^{(2)}$, LÃ THẾ VINH(2) \\ ${ }^{(1)}$ Cuc Đo đạc, Bản đồ và Thông tin địa lý Việt Nam \\ ${ }^{(2)}$ Trường Đại học Bách khoa Hà Nội
}

\section{Tóm tắt:}

Trong bài viết này đề cập đến phưong pháp kiểm định chất luợng phuơng tiện đo định vị bằng vệ tinh (máy thu GNSS) trong phòng dựa trên giải pháp mô phỏng tín hiệu. Đây là một trong nhũng giải pháp có tính ưu việt khi thực hiện kiểm định máy thu GNSS theo chế độ định vị tuyệt đối và định vị đo động thời gian thực RTK. Phuoong pháp này là lựa chọn tối ưu trong việc triển khai thực hiện quản lý chất luợng phưong tiện đo theo huớng đã được quy định trong Luật Đo luờng số 04/2011/QH13 và Luật Đo đạc và bản đồ số 27/2018/QH14

\section{1. Đặt vấn đề}

Kiểm định phương tiện đo trong lĩnh vực đo đạc bản đồ là yêu cầu bắt buộc để đảm bảo phương tiện đo luôn hoạt động ổn định, đạt được độ chính xác theo tiêu chuẩn của nhà sản xuất công bố. Công tác kiểm định phương tiện đo đạc bản đồ là một hoạt động mang tính nguyên tắc không thể thiếu ở mọi quốc gia để đảm bảo độ chính xác hoạt động của phương tiện đo.

Phương tiện đo định vị bằng vệ tinh (máy thu GNSS) đã được đưa vào sử dụng ở Việt Nam từ những năm 1990, đến nay đã phát triển vượt bậc về công nghệ xử lý cũng như chất lượng phương tiện đo; tuy nhiên việc nghiên cứu về các giải pháp kiểm định chất lượng máy thu GNSS ở Việt Nam hiện nay chưa có nhiều. Trước đây máy thu GNSS thuộc phương tiện đo nhóm 1 do vậy việc kiểm định theo yêu cầu của tổ chức, cá nhân và thực hiện theo các quy trình nội bộ của các đơn vị thực hiện công tác kiểm định. Hiện nay theo Thông tư 07/2019/TT-BKHCN ngày 26 tháng 7 năm 2019 thì Phương tiện đo định vị bằng vệ tinh (máy thu GNSS) thuộc phương tiện đo nhóm 2 sẽ phải kiểm định bắt buộc và thực hiện theo quy trình chuẩn do Tổng Cục Tiêu chuẩn Đo lường Chất lượng ban hành.

Nghiên cứu lựa chọn giải pháp tối ưu để thực hiện công tác kiểm định máy thu GNSS theo đúng yêu cầu của Luật Đo lường là cần thiết để đảm bảo công tác kiểm định được thực hiện nghiêm túc, thống nhất, có chất lượng; đảm bảo chất lượng kỹ thuật về đo lường của máy thu GNSS khi đưa vào sản xuất nhằm nâng cao hơn nữa chất lượng các sản phẩm của ngành đo đạc và bản đồ đáp ứng kịp thời cho cuộc cách mạng công nghiệp 4.0.

\section{Phương pháp kiểm định máy thu GNSS}

Cùng với sự phát triển ngày càng mạnh mẽ của công nghệ định vị và dẫn đường sử dụng vệ tinh toàn cầu GNSS (Global Navigation Satelite Systems), số lượng máy thu GNSS cho nhiều mục đích khác nhau trên thị trường cũng ngày càng nhiều. Chính vì thế để đảm bảo được chất lượng của các máy thu GNSS này, các nhà sản xuất cần phải kiểm định theo các tiêu chí khác nhau trong đó đặc biệt quan trọng là tiêu chí về độ chính xác vị trí, thời gian. Mỗi lĩnh vực chuyên môn thường có yêu cầu khác nhau nên

Ngày nhận bài: 5/9/2021, ngày chuyển phản biện: 9/9/2021, ngày chấp nhận phản biện: 15/9/2021, ngày chấp nhận đăng: 18/9/021 
quy định về quy trình kiểm định cũng khác nhau. Trước đây, hầu hết các bài kiểm định được thực hiện trong môi trường thực trên hệ thống các mốc có tọa độ chuẩn, điều này có những khó khăn do tiêu tốn nhiều nguồn lực trong việc xây dựng, duy tu và bảo trì hệ thống mốc chuẩn. Ngoài ra môi trường thực bị ảnh hưởng bởi nhiều yếu tố khách quan như thời tiết, nhiễu từ môi trường xung quanh, nhiễu do bản thân sự cố từ các vệ tinh .v.v. nên việc kiểm định đôi khi không chính xác như mong muốn.

Trước đây, chức năng và ứng dụng của các máy thu GNSS chủ yếu thực hiện các công việc về thành lập lưới tọa độ do vậy khi kiểm định cũng chủ yếu quan tâm đến sai số đo tĩnh. Công tác kiểm định được đo đạc dựa trên các mốc có tọa độ chuẩn, đồng thời phải được tính toán bình sai bằng các phần mềm có độ chính xác cao. Coi các yếu tố chủ quan được loại trừ thì chất lượng công tác kiểm định không những vẫn bị ảnh hưởng bởi các yếu tố khách quan về môi trường trong công tác đo đạc mà còn có yếu tố khách quan về quá trình xử lý tính toán bình sai cũng như chất lượng của phần mềm xử lý. Hiện nay trên thế giới và cả Việt Nam đã và đang phát triển các hệ thống trạm CORS (Continuous Reference Station) để đảm bảo cho công tác định vị dẫn đường và nhiều mục đích khác, khi đó các máy thu GNSS chủ yếu sẽ sử dụng các giải pháp của trạm CORS trong việc dẫn đường và đo động thời gian thực RTK (Real-Time Kinematic), giải pháp đo tĩnh sẽ không còn sử dụng nhiều trong thực tế.

Với các lý do nêu trên nên trong ISO 171238 của Phiên bản đầu 2007-09-15 về "Lĩnh vục Quang hoc và dụng cu quang học, Thủ tục kiểm tra dụng cu khảo sát và trắc địa" cũng chỉ xây dựng quy trình kiểm định máy thu GNSS với giải pháp đo RTK mà không đưa ra giải pháp và quy trình kiểm định về đo tĩnh. Các nghiên cứu trước đây trên thế giới và trong quy trình ISO 17123-8 cũng xây dựng cách thức và giải pháp kiểm định máy thu GNSS theo chế độ định vị tuyệt đối và định vị RTK nhưng được thực hiện ngoài hiện trường. Tuy nhiên như đã nói ở trên giải pháp thực hiện ngoài hiện trường không thể loại bỏ được hoàn toàn sự ảnh hưởng của môi trường đến chất lượng công tác kiểm định.

Trong những năm gần đây, giải pháp mô phỏng tín hiệu vệ tinh được nhiều nhà sản xuất máy thu GNSS sử dụng trong kiểm định nhờ ưu điểm tiết kiệm nguồn lực và có thể tái tạo môi trường kiểm định khách quan (có tính lặp lại với mọi lần kiểm định khác nhau). Tuy nhiên để sử dụng giải pháp mô phỏng tín hiệu trong kiểm định máy thu GNSS cũng cần có những kiến thức và quy trình thực hiện chính xác.

\section{Bộ mô phỏng tín hiệu}

Hệ thống dẫn đường vệ tinh toàn cầu GNSS là thuật ngữ chung để chỉ một hệ thống có khả năng cung cấp thông tin dẫn đường và các dịch vụ khác cho người dùng trên khắp thế giới. Mỗi hệ thống GNSS đều sở hữu một chùm các vệ tinh, các vệ tinh truyền phát những tín hiệu theo chuẩn riêng, các tín hiệu này sau đó sẽ được xử lý bởi các máy thu GNSS nhằm xác định vị trí, vận tốc và thời gian, phục vụ cho người sử dụng ở bất kỳ đâu trên bề mặt trái đất. Các hệ thống GNSS đang hoạt động và tiếp tục được phát triển hiện nay bao gồm: Hệ thống GPS (Hoa Kỳ), Hệ thống GLONASS $(\mathrm{Nga})$, Hệ thống Galileo (Châu Âu), Hệ thống Beidou/Compass (Trung Quốc), Hệ thống QZSS (Nhật Bản) và hệ thống IRNSS (Ần Độ). 


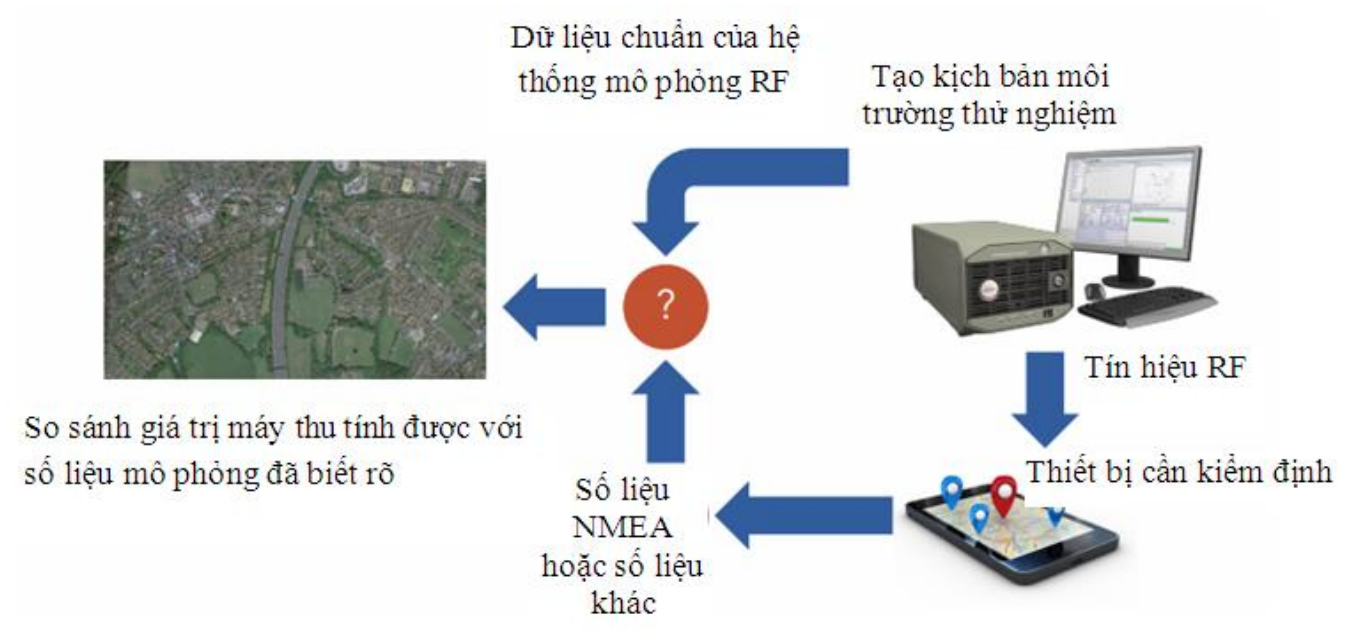

Hình 1: Mô hình hệ thống mô phỏng

Bộ mô phỏng tín hiệu GNSS theo hình 1 gồm hệ thống phần cứng, phần mềm được thiết kế nhằm mục đích cung cấp phương thức kiểm tra thử nghiệm các máy thu GNSS hiệu quả và ý nghĩa nhất, thực tiễn đã cho thấy hiệu quả to lớn của phương thức kiểm tra này. Bộ mô phỏng GNSS tái xác lập môi trường của các máy thu GNSS dựa trên nền tảng động, bằng cách mô hình hoá chuyển động của phương tiện và vệ tinh, các đặc điểm riêng của tín hiệu định vị, khí quyển và các yếu tố ảnh hưởng khác .v.v. tất cả các yếu tố có ảnh hưởng tới máy thu GNSS được ghi nhận trong quá trình hoạt động thực tiễn, dựa vào các tham số đã biết để xây dựng lại khung cảnh môi trường kiểm tra thử nghiệm. Máy thu GNSS sẽ xử lý những tín hiệu được mô phỏng một cách chính xác, giống như chúng nhận được từ các vệ tinh GNSS trong thực tiễn.

Với Bộ mô phỏng GNSS, người kiểm tra có thể dễ dàng tổng hợp và thử nghiệm nhiều kịch bản khác nhau, phục vụ cho các dạng kiểm tra thử nghiệm đặc thù. Trong đó người sử dụng có toàn quyền kiểm soát và điều khiển các yếu tố như: thời điểm mô phỏng, vị trí, quỹ đạo chuyển động của phương tiện, các điều kiện môi trường, các lỗi tín hiệu cũng như mức độ thiếu chính xác của hệ thống. Khi sử dụng Bộ mô phỏng tín hiệu, chúng ta có thể hoàn toàn loại trừ các nguồn sai số do hệ thống và môi trường gây ra như: sai số đồng hồ vệ tinh, sai số quỹ đạo vệ tinh, sai số số liệu dẫn đường, sai số do khí quyển, nhiễu đa đường (Multipath), nhiễu giao thoa.

Các bộ mô phỏng RF (Radio Frequency) của chùm vệ tinh (Constellation Simulator) có khả năng tái tạo lại môi trường để máy thu GNSS hoạt động trên nền tảng động, thông qua mô hình phương tiện và chuyển động của vệ tinh, các đặc tính riêng của tín hiệu định vị, khí quyển và các yếu tố có ảnh hưởng khác .v.v. làm cơ sở để máy thu GNSS có khả năng hoạt động dựa trên tín hiệu RF của các bộ mô phỏng, tuân theo các tham số đã xác lập khi xây dựng cảnh kiểm tra giả định. Có thể các bộ mô phỏng không phải là chiếc hộp thần kỳ để tái tạo lại thế giới thực một cách tuyệt đối, chúng cũng có những hạn chế nhất định. Tuy nhiên vượt xa những hạn chế cố hữu, các bộ mô phỏng vẫn mang đến những lợi ích quan trọng mà không có phương pháp nào có thể thay thế được. Cũng cùng một phương thức như vậy, các kỹ sư thiết kế RF có thể sẽ không sử dụng các bộ tổng hợp nhiễu ngẫu nhiên, khi họ thực sự cần tín hiệu kiểm tra có thể điều khiển và lượng hoá. Những nhà kiểm tra máy thu GNSS chuyên nghiệp cũng có thể không sử dụng đến thiết bị tái tạo tín hiệu thế giới thực ngẫu nhiên, khi họ thực sự cần tín hiệu kiểm tra GNSS có thể điều khiển và mô phỏng lặp lại. 


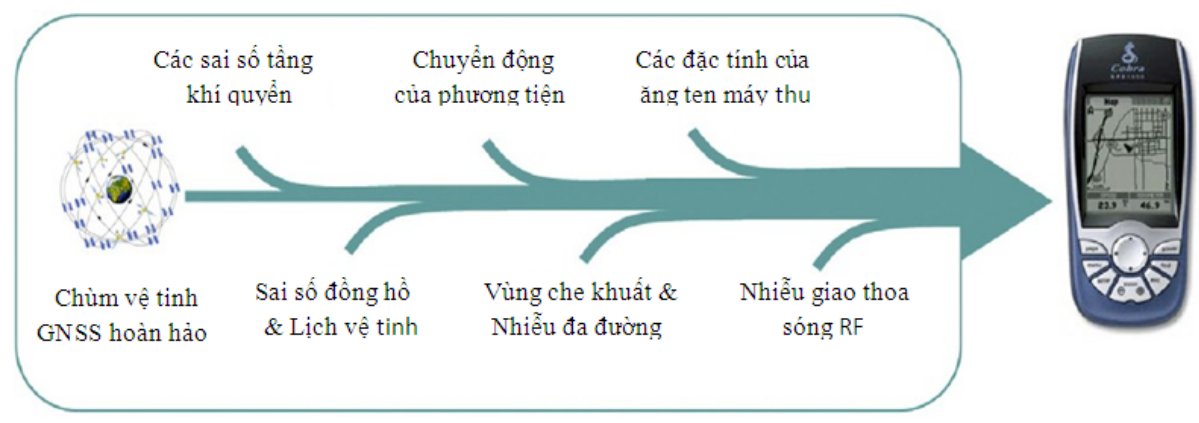

Hình 2: Dòng dịch chuyển của các tín hiệu mô phỏng

Khả năng hoạt động của máy thu GNSS phụ thuộc nhiều vào mức độ nghiêm trọng của các sai số và ảnh hưởng của chúng tới tín hiệu RF. Trong hình 2 thể hiện đường đi của tín hiệu qua một bộ mô phỏng tiêu chuẩn, với những yếu tố tác động được bổ sung, cho tới khi tín hiệu RF cuối cùng được xuất ra, để từ đó tổng hợp tín hiệu RF phức hợp phục vụ cho máy thu GNSS cần kiểm tra. Phương pháp luận này được áp dụng cho tất cả các bộ mô phỏng với một số lượng hữu hạn các yếu tố ảnh hưởng bổ sung thêm, nó phụ thuộc vào khả năng của từng bộ mô phỏng và ứng dụng mà các bộ mô phỏng này hướng tới.

\section{Thực nghiệm kiểm định máy thu GNSS} 4.1. Bộ mô phỏng tín hiệu GNSS NAVISIM
Hiện nay trên thế giới có một số hãng công nghệ sản xuất các sản phẩm phục vụ công tác kiểm định máy thu GNSS dựa trên Bộ mô phỏng tín hiện (VD như: hệ thống NCS NOVA, TeleOrbit của Đức; Spirent của Anh)

Tại Việt Nam, Trung tâm hợp tác nghiên cứu và phát triển công nghệ định vị sử dụng vệ tinh (NAVIS) thuộc trường Đại học Bách khoa Hà Nội đã nghiên cứu và chế tạo thành công Bộ mô phỏng tín hiệu GNSS NAVISIM với rất nhiều chức năng khác nhau trong đó có chức năng dành cho công tác kiểm định máy thu GNSS.

Các thành phần chính của bộ mô phỏng tín hiệu GNSS NAVISIM phục vụ kiểm định được nhóm nghiên cứu như trong Hình 3.

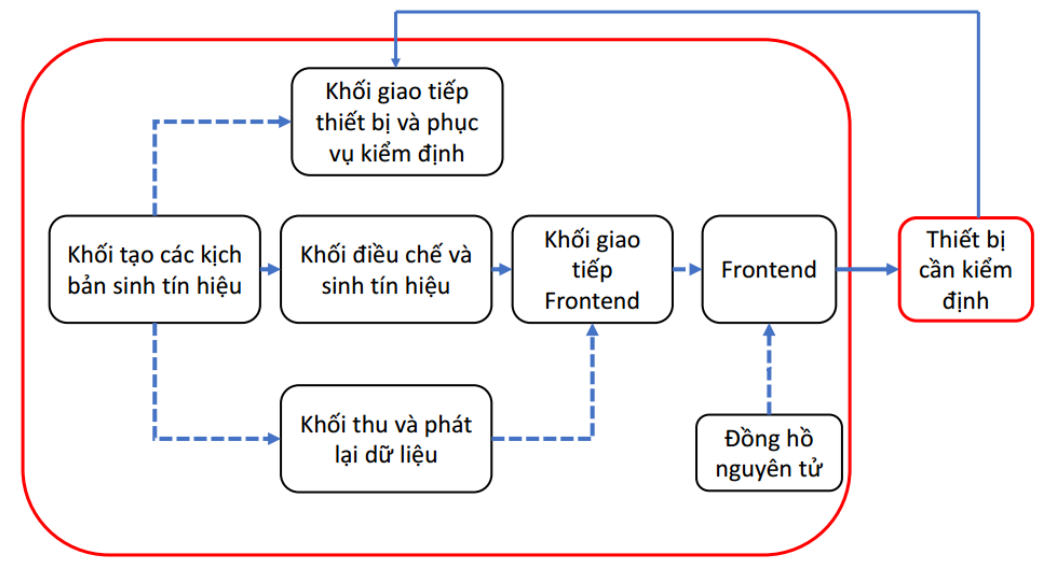

Hình 3: Kiến trúc tổng quan của bộ mô phỏng tín hiệu GNSS NAVISIM

- Khối tạo các kịch bản sinh tín hiệu: Hỗ trợ và tạo các kịch bản kiểm định theo yêu cầu của người dùng. Các kịch bản kiểm định bao gồm thông tin về quỹ đạo di chuyển của người dùng (vị trí, vận tốc và thời gian); các tham số tín hiệu (năng lượng, sai số vệ tinh, sai số đồng hồ ...) và các tham số nhiễu (nhiễu đa đường, nhiễu điện 1y).

- Khối điều chế và sinh tín hiệu: Dựa trên các tham số từ khối kịch bản sinh tín hiệu, khối này sẽ thực hiện điều chế và sinh tín hiệu điều chế dạng số. 


\section{Nghiên cúu}

- Khối giao tiếp Frontend: Khối này sẽ thực hiện truyền dữ liệu tốc độ cao từ khối điều chế và sinh tín hiệu đến Frontend.

- Khối thu và phát lại dũ liệu: Dựa trên kịch bản thu phát lại, khối này sẽ thực hiện việc thu và phát lại dữ liệu. Dũ liệu đầu ra của khối thu và đầu vào của khối phát sẽ là tín hiệu số ở băng cơ sở (Baseband) hoặc trung tần (Intermediate Frequency - IF).

- Khối Frontend: Khối Frontend sẽ thực hiện việc chuyển đổi dữ liệu số từ baseband hoặc IF sang tín hiệu RF.

- Đồng hồ nguyên tư: Tạo dao động có độ chính xác cao để cung cấp cho khối Frontend.

- Khối giao tiếp thiết bị và phục vụ kiểm định: Khối này sẽ thực hiện việc đọc dữ liệu định vị từ thiết bị cần kiểm định, tiến hành so sánh dữ liệu đọc được với dữ liệu từ khối tạo kịch bản sinh dữ liệu.

\subsection{Kết quả thục nghiệm}

a. Tiến hành thực nghiệm

- Bộ mô phỏng tín hiệu: TeleOrbit của Đức.

- Máy GNSS thử nghiệm: Máy Trimble R9 Net (mP:8mm; mH:15mm).

- Nội dung thử nghiệm: Chế độ đo RTK, thu 1 giây một trị đo.

- Số lượng trị đo: 100 trị đo.

- Tín hiệu giả lập: Từ tín hiệu thu thực tế tại khu vực thử nghiệm
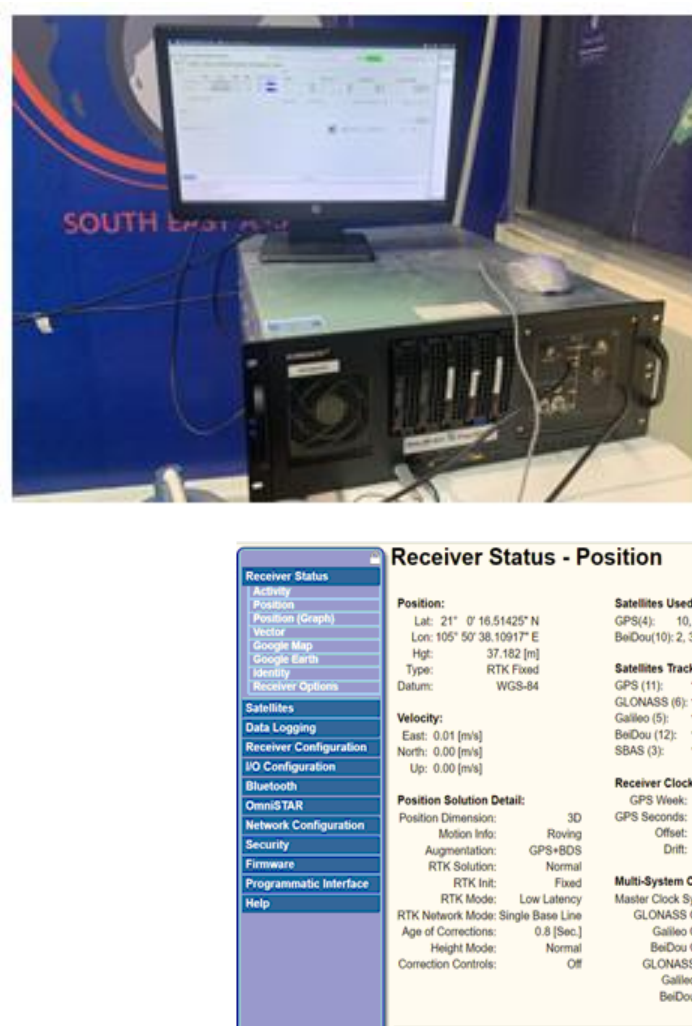
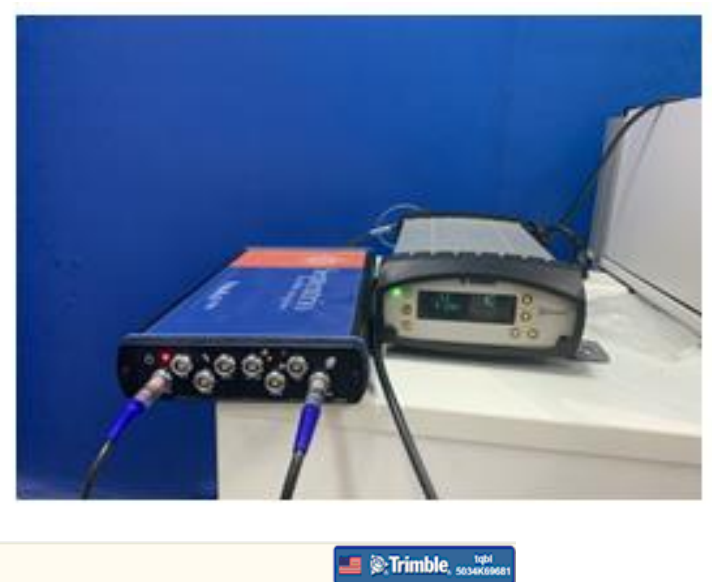

Hình 4: Quá trình thục nghiệm kiểm định máy GNSS

b. Kết quả thưc nghiệm

\begin{tabular}{|c|c|c|c||c|c|c|c|}
\hline TT & B (độ) & L (độ) & H(m) & TT & B (độ) & L (độ) & H(m) \\
\hline 1 & 21.004587325 & 105.843919239 & 37.179 & 51 & 21.004587311 & 105.843919183 & 37.178 \\
\hline 2 & 21.004587322 & 105.843919189 & 37.170 & 52 & 21.004587339 & 105.843919208 & 37.186 \\
\hline 3 & 21.004587325 & 105.843919267 & 37.174 & 53 & 21.004587319 & 105.843919200 & 37.173 \\
\hline 4 & 21.004587303 & 105.843919264 & 37.186 & 54 & 21.004587322 & 105.843919208 & 37.176 \\
\hline
\end{tabular}


Nghiên cúu

\begin{tabular}{|c|c|c|c|c|c|c|c|}
\hline 5 & 21.004587328 & 105.843919200 & 37.179 & 55 & 21.004587311 & 105.843919208 & 37.173 \\
\hline 6 & 21.004587333 & 105.843919192 & 37.182 & 56 & 21.004587314 & 105.843919178 & 37.173 \\
\hline 7 & 21.004587314 & 105.843919219 & 37.182 & 57 & 21.004587292 & 105.843919228 & 37.170 \\
\hline 8 & 21.004587317 & 105.843919186 & 37.176 & 58 & 21.004587311 & 105.843919214 & 37.183 \\
\hline 9 & 21.004587311 & 105.843919211 & 37.179 & 59 & 21.004587328 & 105.843919192 & 37.175 \\
\hline 10 & 21.004587300 & 105.843919203 & 37.187 & 60 & 21.004587333 & 105.843919175 & 37.164 \\
\hline 11 & 21.004587283 & 105.843919208 & 37.188 & 61 & 21.004587322 & 105.843919208 & 37.179 \\
\hline 12 & 21.004587314 & 105.843919178 & 37.176 & 62 & 21.004587286 & 105.843919189 & 37.179 \\
\hline 13 & 21.004587314 & 105.843919236 & 37.173 & 63 & 21.004587325 & 105.843919203 & 37.180 \\
\hline 14 & 21.004587314 & 105.843919186 & 37.179 & 64 & 21.004587317 & 105.843919197 & 37.186 \\
\hline 15 & 21.004587303 & 105.843919194 & 37.182 & 65 & 21.004587283 & 105.843919217 & 37.175 \\
\hline 16 & 21.004587311 & 105.843919186 & 37.177 & 66 & 21.004587331 & 105.843919231 & 37.176 \\
\hline 17 & 21.004587283 & 105.843919203 & 37.186 & 67 & 21.004587339 & 105.843919219 & 37.173 \\
\hline 18 & 21.004587281 & 105.8 & 37.179 & 68 & 21.004 & 105.843919208 & 37.180 \\
\hline 19 & 21.004587317 & 105.843919189 & 37.174 & 69 & 21.004587311 & 105.843919181 & 37.169 \\
\hline 20 & 21.00458 & 105.8 & 37.183 & 70 & 21.00 & 19139 & 37.171 \\
\hline 21 & 21.004587292 & 105.843919183 & 37.176 & 71 & 21.004587331 & 105.843 & 37.176 \\
\hline 22 & 21.004587311 & 105.843919206 & 37.170 & 72 & 21.0045 & 105.8 & 37.174 \\
\hline 23 & 21.004587283 & 105.843919233 & 37.171 & 73 & 21.004587314 & 105.843919200 & 37.177 \\
\hline 24 & 21.004587294 & 105.843919236 & 37.178 & 74 & 21.00458 & 105. & 37.171 \\
\hline 25 & 21.004587311 & 105.843919214 & 37.179 & 75 & 21.004587319 & 105.8439 & 37.179 \\
\hline 26 & 21.004587300 & 105.843919225 & 37.175 & 76 & 21.004587333 & 105.843919228 & 37.168 \\
\hline 27 & 21.004 & 105.8 & 37.174 & 77 & 21.00 & 105.8 & 37.172 \\
\hline 28 & 21.004587300 & 105.843919250 & 37.169 & 78 & 21.00458 & 105. & 37.179 \\
\hline 29 & 21.004587286 & 105.8 & 37.171 & 79 & 21.004587322 & 105.84391 & 37.167 \\
\hline 30 & 21.004587339 & 105.8 & 37.172 & 80 & 21.00 & 105. & 37.179 \\
\hline 31 & 21.004587314 & 105.8 & 37.174 & 81 & 21.00 & 105.8 & 37.192 \\
\hline 32 & 21.004 & 105.8 & 37.177 & 82 & 21.004587311 & 105.8 & 37.172 \\
\hline 33 & 21.004587308 & 105.843919217 & 37.180 & 83 & 21.004587297 & 105.843919253 & 37.174 \\
\hline 34 & 21.004587308 & 105.843919250 & 37.176 & 84 & 21.004587308 & 105.843919175 & 37.188 \\
\hline 35 & 21.004587314 & 105.843919197 & 37.169 & 85 & 21.004587314 & 105.843919178 & 37.173 \\
\hline 36 & 21.004587292 & 105.843919211 & 37.179 & 86 & 21.004587308 & 105.843919206 & 37.182 \\
\hline 37 & 21.004587283 & 105.843919186 & 37.183 & 87 & 21.004587331 & 105.843919219 & 37.177 \\
\hline 38 & 21.004587314 & 105.843919239 & 37.176 & 88 & 21.004587322 & 105.843919183 & 37.170 \\
\hline 39 & 21.004587297 & 105.843919200 & 37.172 & 89 & 21.004587311 & 105.843919228 & 37.186 \\
\hline 40 & 21.004587319 & 105.843919203 & 37.173 & 90 & 21.004587339 & 105.843919211 & 37.181 \\
\hline 41 & 21.004587328 & 105.843919244 & 37.180 & 91 & 21.004587314 & 105.843919192 & 37.180 \\
\hline 42 & 21.004587289 & 105.843919197 & 37.175 & 92 & 21.004587339 & 105.843919150 & 37.175 \\
\hline 43 & 21.004587306 & 105.843919236 & 37.183 & 93 & 21.004587314 & 105.843919247 & 37.171 \\
\hline 44 & 21.004587283 & 105.843919217 & 37.170 & 94 & 21.004587314 & 105.843919225 & 37.182 \\
\hline 45 & 21.004587314 & 105.843919200 & 37.184 & 95 & 21.004587303 & 105.843919267 & 37.174 \\
\hline 46 & 21.004587317 & 105.843919194 & 37.173 & 96 & 21.004587300 & 105.843919178 & 37.177 \\
\hline 47 & 21.004587339 & 105.843919239 & 37.183 & 97 & 21.004587300 & 105.843919211 & 37.180 \\
\hline 48 & 21.004587342 & 105.843919186 & 37.168 & 98 & 21.004587292 & 105.843919214 & 37.182 \\
\hline 49 & 21.004587317 & 105.843919236 & 37.169 & 99 & 21.004587325 & 105.843919219 & 37.183 \\
\hline 50 & 21.004587347 & 105.843919219 & 37.182 & 100 & 21.004587339 & 105.843919250 & 37.175 \\
\hline
\end{tabular}




\section{Nghiên cúu}

- Sai lệch giữa trị lớn nhất và nhỏ nhất $(\Delta \mathrm{x}=0.009 \mathrm{~m} ; \Delta \mathrm{y}=0.014 \mathrm{~m} ; \Delta \mathrm{H}=0.028 \mathrm{~m})$

- Sai số máy thu GNSS (tính theo công thức Bessen): $(\mathrm{mP}=0.003 \mathrm{~m} ; \mathrm{mH}=0.005 \mathrm{~m})$

- Kết quả kiểm định: Chất lượng máy thu GNSS đạt yêu cầu theo catalog.

\section{Kết luận}

Qua nghiên cứu nhận thấy công tác kiểm định máy thu GNSS sử dụng Bộ mô phỏng tín hiệu là một giải pháp ưu việt cho quá trình thực hiện công tác kiểm định cũng như đảm bảo chất lượng của quá trình kiểm định.

Với đặc tính ưu việt của phương pháp cũng như việc Việt Nam đã có những nghiên cứu và chế tạo thành công thiết bị mô phỏng tín hiệu thì giải pháp kiểm định này cần được triển khai và áp dụng rộng rãi trong thực tế nhằm đưa công tác kiểm định phương tiện đo đạc nói chung và máy thu GNSS nói riêng được thống nhất, chuẩn chỉ theo các yêu cầu của Luật Đo lường và Luật Đo đạc và bản đồ, góp phần đảm bảo tiêu chuẩn chất lượng các sản phẩm đo đạc bản đồ - một nền tảng cơ bản để xây dựng, đưa nền kinh tế số của Việt Nam phát triển, hội nhập với các quốc gia trong khu vực và trên thế giới. $\bigcirc$

\section{Tài liệu tham khảo}

[1]. ISO 71723-8 Optics and optical instruments, Field procedures for testing geodetic and surveying instruments; part 8: GNSS field measurement systems in real-time kinematic (RTK).

[2]. Luật Đo lường số 04/2011/QH13.

[3]. Luật Đo đạc và bản đồ số 27/2018/QH14.

[4]. Thông tư số 07/2019/TT-BKHCN ngày 26 tháng 7 năm 2019 Sửa đổi, bổ sung một số điều của Thông tư số 23/2013/TT-BKHCN ngày 26 tháng 9 năm 2013 của Bộ trưởng Bộ Khoa học Công nghệ quy định về đo lường đối với phương tiện nhóm $2 . \bigcirc$

\section{Summary}

\section{Research on GNSS receiver verification method based on signal simulation solution}

Bui Dang Quang

Department of Survey, Mapping and Geographic information Vietnam

Nguyen Dinh Thuan

HaNoi University of Science and Technology

La The Vinh

\section{HaNoi University of Science and Technology}

This article discusses on the verification method of GNSS receiver in the room based on signal simulation solution. This is one of the preeminent solutions when verifies GNSS receiver in absolute position mode and RTK. This method is the optimal choice in implementing the quality management of measuring devices in the direction specified in the Law on Measurement No. 04/2011/QH13 and Law on Survey and Mapping No. 27/2018/QH14. O 